\title{
Closing the Brief Case: Disseminated Neisseria gonorrhoeae in an 18-Year-Old Female
}

\author{
Julianne E. Burns, ${ }^{\text {a Erin H. Grafb,c }}$ \\ aDivision of Infectious Diseases, Department of Pediatrics, Children's Hospital of Philadelphia, Philadelphia, \\ Pennsylvania, USA \\ bDepartment of Pathology and Laboratory Medicine, Children's Hospital of Philadelphia, Philadelphia, \\ Pennsylvania, USA \\ 'Department of Pathology and Laboratory Medicine, Perelman School of Medicine, University of Pennsylvania, \\ Philadelphia, Pennsylvania, USA
}

KEYWORDS disseminated gonococcal infection, Neisseria gonorrhoeae, septic arthritis

\section{SELF ASSESSMENT QUESTIONS}

1. Which of the following specimens is recommended and FDA cleared for nucleic acid amplification testing (NAAT) in suspected cases of disseminated Neisseria gonorrhoeae?
A. Whole blood
B. Urine
C. Synovial fluid
D. Serum

Answer: B. While patients with disseminated gonococcal infection may be bacteremic, testing of whole blood or plasma for $N$. gonorrhoeae nucleic acids is insensitive. A few studies have shown positive $N$. gonorrhoeae PCR results from joint fluid in cases of gonococcal arthritis, but this source is not FDA cleared on any N. gonorrhoeae NAAT platforms. Urine is the only source from the list that is FDA cleared and recommended for $N$. gonorrhoeae NAAT testing. A positive result from urine is considered supportive evidence of disseminated gonococcal infection in the right clinical setting.

2. What is the estimated sensitivity of aerobic culture for the detection of $N$. gonorrhoeae in synovial fluid?
A. $\sim 10 \%$
B. $\sim 25 \%$
C. $\sim 50 \%$
D. $\sim 90 \%$

Answer: C. Culture of synovial fluid the from the affected joint is insensitive for recovery of $N$. gonorrhoeae. It is thought that the synovial fluid itself may be inhibitory to bacterial growth. Nonetheless, culture is still important to isolate colonies for susceptibility testing. Thus, culture should be performed from aspirated joint fluid in cases of gonococcal arthritis.

3. Which of the following is the recommended treatment for disseminated gonococcal infection?

A. Cefpodoxime plus azithromycin

B. Ceftriaxone plus doxycycline

Citation Burns JE, Graf EH. 2018. Closing the Brief Case: Disseminated Neisseria gonorrhoeae in an 18-year-old female. J Clin Microbiol 56:e00933-17. https://doi.org/10.1128/JCM .00933-17.

Editor Carey-Ann D. Burnham, Washington University School of Medicine Copyright $\odot 2018$ American Society for Microbiology. All Rights Reserved. Address correspondence to Erin H. Graf, grafe@email.chop.edu.

See https://doi.org/10.1128/JCM.00932-17 in this issue for case presentation and discussion. 
C. Cefixime plus doxycycline

D. Ceftriaxone plus azithromycin

Answer: D. All treatment combinations listed other than ceftriaxone plus azithromycin are alternative regimens. If susceptibility to either cefpodoxime or cefixime can be demonstrated, these oral 3rd-generation cephalosporins may be used. In the case of an allergy to azithromycin, doxycycline can be substituted.

\section{TAKE HOME POINTS}

- Gonococcal arthritis can be diagnosed through Gram staining and culture of synovial fluid, though both have very limited sensitivity.

- There are no commercially available molecular tests for Neisseria gonorrhoeae from synovial fluid. Molecular testing from urogenital sources and/or rectal and pharyngeal sources, if validated, offers a supportive diagnosis when cultures of joint fluid are negative.

- Culture of Neisseria gonorrhoeae is still important for antimicrobial susceptibility testing, particularly in cases of disseminated infection. 